\title{
Oblique Derivative Problems for Elliptic Systems of Second Order Equations in Infinite Domains
}

\author{
H. Begehr and G. C. Wen
}

Dedicated to Prof. Dr. L. von Wolfersdorf on the occasion of his 65th birthday

\begin{abstract}
There are many problems in mechanics and physics, the mathematical models of which are some boundary value problems for nonlinear elliptic systems of first and second order equations in multiply connected domains including infinity. In this paper, we discuss oblique derivative problems for systems of second order equations.
\end{abstract}

Keywords: Complex partial differential equations, oblique derivative problems

AMS subject classification: $35 \mathrm{~J} 25,35 \mathrm{~J} 60,35 \mathrm{~J} 65$

\section{Formulation of the problems}

Let $D$ be an $(N+1)$-connected domain in $\mathbb{C}$ including infinity, with boundary $\Gamma=$ $\cup_{j=0}^{N} \Gamma_{j} \in C_{\alpha}^{2} \quad(0<\alpha<1)$. Without loss of generality we may assume that $D$ is a circular domain in $\{z \in \mathbb{C}:|z|>1\}$, whose boundary consists of $N+1$ circles $\Gamma_{0}=\Gamma_{N+1}=\{z \in \mathbb{C}:|z|=1\}$ and $\Gamma_{j}=\left\{z \in \mathbb{C}:\left|z-z_{j}\right|=\gamma_{j}\right\} \quad(j=1, \ldots, N)$, where $z_{j} \in \mathbb{C}$ are given points, $0<\gamma_{j} \in \mathbb{R}$ are given constants (sce, e.g., $[2,3]$ ).

We consider the nonlinear elliptic system of second order equations in complex form

$$
\left.\begin{array}{rl}
w_{z \bar{z}} & =F\left(z, w, w_{z}, \bar{w}_{z}, w_{z z}, \bar{w}_{z z}\right) \\
F & =Q_{1} w_{z z}+Q_{2} \bar{w}_{z z}+A_{1} w_{z}+A_{2} \bar{w}_{z}+A_{3} w+A_{4} \\
Q_{j} & =Q_{j}\left(z, w, w_{z}, \bar{w}_{z}, w_{z z}, \bar{w}_{z z}\right) \quad(j=1,2) \\
A_{j} & =\dot{A}_{j}\left(z, w, w_{z}, \bar{w}_{z}\right) \quad(j=1,2,3,4) .
\end{array}\right\}
$$

Suppose that (1.1) satisfies the following conditions $(C)_{1}-(C)_{3}$.

(C) $Q_{j}\left(z, w, w_{z}, \bar{w}_{z}, U, V\right)$ and $A_{j}\left(z, w, w_{z}, \bar{w}_{z}\right)$ are continuous in $w, w_{z}, \bar{w}_{z} \in \mathbb{C}$ for almost every $z \in D$ and all $U, V \in \mathbb{C}$, and $Q_{j}=0$ and $A_{j}=0$ for $z \notin D$.

H. Begehr: Freie Univ. Berlin, I. Math. Inst., Arnimallee 3, D-14195 Berlin

G. C. Wen: Peking University, Dept. Math., Beijing 100871, P. R. China. This research was carried out while the author was visiting FU Berlin on the basis of a DAAD-K.C. Wong Fellowship during March to August 1997. 
(C) $Q_{2} Q_{j}\left(z, w, w_{z}, \bar{w}_{z}, U, V\right)$ and $A_{j}\left(z, w, w_{z}, \bar{w}_{z}\right)$ are measurable in $z \in D$ for all continuously differentiable functions $w=w(z)$ on $\bar{D}$ and all measurable functions $U, V \in L_{p_{0}, 2}(\bar{D})$, and satisfy

$$
L_{p, 2}\left[A_{j}\left(z, w, w_{z}, \bar{w}_{z}\right), \bar{D}\right] \leq k_{j-i} \quad(j=1, \ldots, 4)
$$

in which $p_{0}$ and $p$ with $2<p_{0} \leq p$ and $k_{j}(j=0,1,2,3)$ are non-negative constants.

(C) $)_{3}$ System (1.1) satisfies for any functions $w \in C^{1}(\bar{D})$ and constants $U^{j}, V^{j} \in$ $\mathbb{C}(j=1,2)$ the uniform ellipticity condition

$$
\begin{gathered}
\left|F\left(z, w, w_{z}, \bar{w}_{z}, U^{1}, V^{1}\right)-F\left(z, w, w_{z}, \bar{w}_{z}, U^{2}, V^{2}\right)\right| \\
\leq q_{1}\left|U^{1}-U^{2}\right|+q_{2}\left|V^{1}-V^{2}\right|
\end{gathered}
$$

for almost every point $z \in D$, where $q_{1} \geq 0$ and $q_{2} \geq 0$ are constants with $q_{1}+q_{2}<1$.

Now we formulate the oblique derivative problem, i.e. the Poincaré boundary value problem as follows (compare [5]).

Problem (P). In the domain $D$, find a solution $w=w(z)$ of system (1.1), which is continuously differentiable on $\bar{D}$ and satisfies the boundary condition

$$
\left.\begin{array}{l}
\operatorname{Re}\left[\overline{\lambda_{1}(z)} w_{z}+a_{11}(z) w\right]=a_{12}(z) \\
\operatorname{Re}\left[\overline{\lambda_{2}(z)} w_{\bar{z}}+a_{21}(z) w\right]=a_{22}(z)
\end{array}\right\} \quad(z \in \Gamma)
$$

where $\lambda_{j}$ with $\left|\lambda_{j}(z)\right|=1$ and $a_{j k}(j, k=1,2)$ are known functions, which satisfy the conditions.

$$
C_{\alpha}\left[\lambda_{j}, \Gamma\right] \leq k_{0}, \quad C_{\alpha}\left[a_{j 1}, \Gamma\right] \leq k_{1}, \quad C_{\alpha}\left[a_{j 2}, \Gamma\right] \leq k_{4}
$$

in which $\alpha$ with $\frac{1}{2}<\alpha<1$ and $k_{0}, k_{1}, k_{4}$ are non-negative constants.

Denote

$$
K_{j}=\frac{1}{2 \pi} \Delta_{Y} \arg \lambda_{j}(z) \quad(j=1,2) .
$$

$\left[K_{1}, K_{2}\right]$ is called the index of Problem (P). When $K_{1}<0$ and $K_{2}<0$, then Problem (P) may not be solvable. Further, when $K_{1} \geq 0$. and $K_{2} \geq 0$, then the solution of Problem $(P)$ is not necessarily unique. Hence we consider the well-posedness of Problem (P) with modified boundary conditions (see $[1,4]$ ).

Problem (Q). Find a continuous solution $[w, U, V]$ of the complex system

$$
\left.\begin{array}{rl}
U_{\bar{z}} & =F\left(z, w, U, V, U_{z}, V_{z}\right) \\
F & =Q_{1} U_{z}+Q_{2} V_{z}+A_{1} U+A_{2} V+A_{3} w+A_{4} \\
V_{\bar{z}} & =\bar{U}_{z}
\end{array}\right\}
$$

satisfying the boundary condition

$$
\operatorname{Re}\left[\overline{\lambda_{j}(z)} U_{j}(z)+a_{j_{1}}(z) w(z)\right]=a_{j 2}(z)+h_{j}(z) \quad(j=1,2 ; z \in \Gamma)
$$


and the relation

$$
w(z)=-\int_{1}^{z}\left[\frac{U(\zeta)}{\zeta^{2}} d \zeta+\frac{\overline{V(\zeta)}}{\bar{\zeta}^{2}} d \bar{\zeta}-\sum_{m=1}^{N} \frac{d_{m} z_{m}}{\zeta\left(\zeta-z_{m}\right)} d \zeta\right]+c_{0}
$$

where $U_{1}=U, U_{2}=V$ and $d_{m}$ are appropriate real constants such that the function determined by the integral in (1.9) is single-valued in $D$, and the undetermined functions $h_{j}$ are of the form

$$
\begin{aligned}
& h_{j}(z)= \\
& \left\{\begin{array}{lll}
0 & \text { if } z \in \Gamma \quad\left(K_{j} \geq N\right) \\
h_{j k} & \text { if } z \in \Gamma_{k}\left(\begin{array}{l}
k=1, \ldots, N-K_{j} \\
0 \leq K_{j}<N
\end{array}\right) \\
0 & \text { if } z \in \Gamma_{k}\left(\begin{array}{l}
k=N-K_{j}+1, \ldots, N+1 \\
0 \leq K_{j}<N
\end{array}\right) \\
h_{j k} & \text { if } z \in \Gamma_{k}\left(\begin{array}{l}
k=1, \ldots, N \\
K_{j}<0
\end{array}\right) \\
h_{j 0}+\operatorname{Re} \sum_{m=1}^{-K_{j-1}\left(h_{j m}^{+}+i h_{j m}^{-}\right) z^{m}} & \text { if } z \in \Gamma_{0} \quad\left(\begin{array}{l}
\left(K_{j}<0\right),
\end{array}\right.
\end{array}\right.
\end{aligned}
$$

Here $h_{j k}$ and $h_{j m}^{ \pm}$are unknown real constants to be determined appropriately. In addition, for $K_{j} \geq 0(j=1,2)$ the solution $w$ is assumed to satisfy the point conditions

$$
\begin{aligned}
& \operatorname{Im}\left[\overline{\lambda_{j}\left(a_{k}\right)} U_{j}\left(a_{k}\right)+a_{j 1} w\left(a_{k}\right)\right]=b_{j k} \quad\left(j=1,2 ; k \in J_{j}\right) \\
& J_{j}=\left\{\begin{array}{ll}
\left\{1, \ldots, 2 K_{j}-N+1\right\} & \text { if } K_{j} \geq N \\
\left\{N-K_{j}+1, \ldots, N+1\right\} & \text { if } 0 \leq K_{j}<N
\end{array}\right\}
\end{aligned}
$$

where $a_{k} \in \Gamma_{k} \quad(k=1, \ldots, N)$ and $a_{k} \in \Gamma_{0} \quad\left(k=N+1, \ldots, 2 K_{j}-N+1\right.$, with $K_{j} \geq$ $N$ for $j=1,2)$ are distinct points and $b_{j k}$ are all real constants satisfying the conditions

$$
\sum_{j=1,2 ; k \in J_{j}}\left|b_{j k}\right| \leq k_{5}
$$

with a non-negative constant $k_{5}$ such that $\left|c_{0}\right| \leq k_{5}$

Problem (Q) $)_{0}$ This is a special case of Problem (Q), namely with $A_{4}=0, a_{j 2}=0$, $b_{j k}=0\left(j=1,2 ; k \in J_{j}\right)$ and $c_{0}=0$.

In order to prove the uniqueness of solutions for Problem $(Q)$, we need to add the condition that for any functions $U^{j}, V^{j}, w^{j} \in \widetilde{C}(\bar{D})(j=1,2)$ with $U_{z}^{1}, V_{z}^{1} \in L_{p_{0}, 2}(\bar{D})$ the equality

$$
\begin{aligned}
& F\left(z, w^{1}, U^{1}, V^{1}, U_{z}^{1}, V_{z}^{1}\right)-F\left(z, w^{2}, U^{2}, V^{2}, U_{z}^{1}, V_{z}^{1}\right) \\
& =\tilde{A}_{1}\left(U^{1}-U^{2}\right)+\tilde{A}_{2}\left(V^{1}-V^{2}\right)+\tilde{A}_{3}\left(w^{1}-w^{2}\right)
\end{aligned}
$$

holds in almost every point $z \in D$, where $L_{p_{0}, 2}\left[\tilde{A}_{j}, \bar{D}\right]<\infty \quad(j=1,2,3)$. 


\section{A priori estimates for solutions of problem (Q)}

In oder to prove the solvability of Problem (Q), we need to give some estimates of its solutions.

Theorem 2.1. Suppose that Conditions $(\mathrm{C})_{1}-(\mathrm{C})_{3}$ hold and the constants $q_{2}$ and $k_{1}, k_{2}$ in (1.2), (1.3) and (1.5) are small enough. Then any solution $[w, U, V]$ of Problem (Q) satisfies the estimates

$$
\left.\begin{array}{l}
L_{1}=L(U)=C_{\beta}[U, \bar{D}]+L_{p_{0}, 2}\left[\left|U_{\bar{z}}\right|+\left|U_{z}\right|, \bar{D}\right] \leq M_{1} \\
L_{2}=L(V) \leq M_{1}
\end{array}\right\}
$$

and

$$
S=S(w)=C_{\beta}^{1}[w, \bar{D}]+L_{p_{0}, 2}\left[\left|w_{z z}\right|+\left|w_{z z}\right|+\left|\bar{w}_{z z}\right|, \bar{D}\right] \leq M_{2}
$$

where $\beta=\min \left(\alpha, 1-\frac{2}{p_{0}}\right), p_{0}$ with $2<p_{0} \leq p, M_{j}=M_{j}\left(q_{0}, p_{0}, k, \alpha, K, D\right) \quad(j=$ 1,$\left.2 ; k=\left(k_{0}, \ldots, k_{5}\right)\right)$ are non-negative constants and $K=\left(K_{1}, K_{2}\right)$.

Proof. Let the solution $[w, U, V]$ of Problem (Q) be substituted into system (1.7), the boundary conditions (1.8) and (1.11), and relation (1.9). It is clear that (1.7) and (1.8) can be rewritten in the form

$$
\left.\begin{array}{l}
U_{\bar{z}}-Q_{1} U_{z}-A_{1} U=A \\
A=Q_{2} V_{z}+A_{2} V+A_{3} w+A_{4} \\
U_{\bar{z}}=\bar{V}_{z}
\end{array}\right\} \quad \text { in } D
$$

and

$$
\left.\begin{array}{l}
\operatorname{Re}\left[\overline{\lambda_{j}(z)} U_{j}(z)\right]=r_{j}(z)+h_{j}(z) \\
\quad \text { with } r_{j}(z)=a_{j 2}(z)-\operatorname{Re}\left[a_{j 1}(z) w(z)\right] \\
\operatorname{Im}\left[\overline{\lambda_{j}\left(a_{k}\right)} U_{j}\left(a_{k}\right)\right]=s_{j k} \\
\quad \text { with } s_{j k}=b_{j k}-\operatorname{Im}\left[\overline{\lambda_{j}\left(a_{k}\right)}\left(a_{k}\right)\right]
\end{array}\right\} \quad\left(j=1,2 ; k \in J_{j} ; z \in \Gamma\right)
$$

where $A$ and $r_{j}, s_{j k}$ satisfy the inequalities

$$
\begin{aligned}
L_{p_{0}, 2}[A, \bar{D}] \leq & q_{2} L_{p_{0}, 2}\left[V_{z}, \bar{D}\right]+L_{p_{0}, 2}\left[A_{2}, \bar{D}\right] C[V, \bar{D}] \\
& +L_{p_{0}, 2}\left[A_{3}, \bar{D}\right] C[w, \bar{D}]+L_{p_{0}, 2}\left[A_{4}, \bar{D}\right] \\
\leq & q_{2} L_{2}+k_{1} L_{2}+k_{2} S_{1}+k_{3}
\end{aligned}
$$

and

$$
\left.\begin{array}{rl}
C_{\alpha}\left[r_{j}, \Gamma\right] & \leq C_{\alpha}\left[a_{j 1}, \Gamma|C| w, \Gamma\right]+C_{\alpha}\left[a_{j 2}, \Gamma\right] \leq k_{1} S_{1}+k_{4} \\
\left|s_{j k}\right| & \leq k_{1} S_{1}+k_{5}
\end{array}\right\} \quad\left(j=1,2 ; k \in J_{j}\right)
$$


in which $\left.S_{1}=C \mid w, \bar{D}\right]$. In accordance with the estimates on Problem $B$ for (2.3) in [4], we obtain

$$
\begin{aligned}
L_{1} & \leq M_{3}\left[\left(q_{2}+k_{1}\right) L_{2}+k_{2} S_{1}+k_{3}+2 k_{1} S_{1}+k_{4}+k_{5}\right] \\
& =M_{3}\left[\left(q_{2}+k_{1}\right) L_{2}+\left(k_{2}+2 k_{1}\right) S_{1}+k_{3}+k_{4}+k_{5}\right]
\end{aligned}
$$

where $M_{3}=M_{3}\left(q_{0}, p_{0}, k, \alpha, K, D\right)$. Moreover, noting that $V$ is a solution of the modified Riemann-Hilbert problem for $U_{\bar{z}}=\bar{V}_{z}$, we have

$$
L_{2} \leq M_{3}\left[L_{1}+2 k_{1} S_{1}+k_{4}+k_{5}\right] \text {. }
$$

In addition, from (1.9) it can be derived that

$$
S_{1}=C[w, \bar{D}] \leq k_{5}+M_{4}[C(U, \bar{D})+C(V, \bar{D})] \leq k_{5}+M_{4}\left(L_{1}+L_{2}\right) .
$$

where $M_{4}=M_{4}(D)$. Combining (2.7) - (2.9), it is derived that

$$
\begin{aligned}
L_{2} \leq & M_{3}\left\{M_{3}\left[\left(q_{2}+k_{1}\right) L_{2}+\left(k_{2}+2 k_{1}\right)\left(k_{5}+M_{4}\left(L_{1}+L_{2}\right)\right)+k_{3}+k_{4}+k_{5}\right]\right. \\
& \left.+2 k_{1}\left(k_{5}+M_{4}\left(L_{1}+L_{2}\right)\right)+k_{4}+k_{5}\right\} \\
\leq & M_{3}\left\{\left(q_{2}+k_{1}\right) M_{3} L_{2}+\left(k_{2}+2 k_{1}\right)\left(1+M_{3}\right) M_{4}\left(L_{1}+L_{2}\right)\right. \\
& \left.+k_{5}\left(k_{2}+2 k_{1}\right)\left(1+M_{3}\right)+\left(k_{3}+k_{4}+k_{5}\right)\left(1+M_{3}\right)\right\}
\end{aligned}
$$

Provided that the constants $q_{2}$ and $k_{1}, k_{2}$ are sufficiently small, for instance, when

$$
M_{3}\left[\left(q_{2}+k_{1}\right) M_{3}+\left(k_{2}+2 k_{1}\right)\left(1+M_{3}\right) M_{4}\right]<\frac{1}{2},
$$

we thus have

$$
\begin{aligned}
L_{2} \leq & 2 M_{3}\left[\left(k_{2}+2 k_{1}\right)\left(1+M_{3}\right) M_{4} L_{1}\right. \\
& \left.+k_{5}\left(k_{2}+2 k_{1}\right)\left(1+M_{3}\right)+\left(k_{3}+k_{4}+k_{5}\right)\left(1+M_{3}\right)\right] \\
= & M_{5} L_{1}+M_{6} .
\end{aligned}
$$

Substituting (2.11) and (2.9) into (2.7), it can be obtained that

$$
\begin{aligned}
L_{1} \leq & M_{3}\left[\left(q_{2}+k_{1}\right)\left(M_{5} L_{1}+M_{6}\right)+\left(k_{2}+2 k_{1}\right) M_{4}\left(L_{1}+L_{2}\right)\right. \\
& \left.+k_{5}\left(k_{2}+2 k_{1}\right)+k_{3}+k_{4}+k_{5}\right] \\
\leq & M_{3}\left\{\left[\left(q_{2}+k_{1}\right) M_{5}+\left(k_{2}+2 k_{1}\right) M_{4}\left(1+M_{5}\right)\right] L_{1} .\right. \\
& \left.+\left(q_{2}+k_{1}\right) M_{6}+\left(k_{2}+2 k_{1}\right) M_{4} M_{6}+k_{5}\left(k_{2}+2 k_{1}\right)+k_{3}+k_{4}+k_{5}\right\} .
\end{aligned}
$$


Moreover, choose $q_{2}$ and $k_{1}, k_{2}$ small enough such that

$$
M_{3}\left[\left(q_{2}+k_{1}\right) M_{5}+\left(k_{2}+2 k_{1}\right)\left(1+M_{5}\right) M_{4}\right]<\frac{1}{2} .
$$

Then it can be concluded that

$$
\begin{aligned}
L_{1} & \leq 2 M_{3}\left[\left(q_{2}+k_{1}\right) M_{6}+\left(k_{2}+2 k_{1}\right) M_{4} M_{6}+k_{5}\left(k_{2}+2 k_{1}\right)+k_{3}+k_{4}+k_{5}\right] \\
& =M_{7}
\end{aligned}
$$

and

$$
L_{2} \leq M_{5} M_{7}+M_{6} \leq M_{1}=\max \left(M_{7}, M_{5} M_{7}+M_{6}\right) .
$$

Furthermore, from (1.9) it follows that (2.2) holds

From Theorem 2.1 we can derive the following result.

Theorem 2.2. Under the conditions of Theorem 2.1, any solution $[w, U, V]$ of Problem (Q) satisfies the estimates

$$
\left.\begin{array}{l}
L_{1}=L(U) \leq M_{8} k^{*} \\
L_{2}=L(V) \leq M_{8} k^{*}
\end{array}\right\}
$$

and

$$
S=S(w) \leq M_{9} k^{*}
$$

where $k^{*}=k_{3}+k_{4}+k_{5}$ and $M_{j}=M_{j}\left(q_{0}, p_{0}, k_{0}, \alpha, K, D\right) \quad(j=8,9)$.

Proof. If $k^{*}=0$, i.e. $k_{3}=k_{4}=k_{5}=0$, the estimates in (2.15) can be derived by Theorem 3.1 below. If $k^{*}>0$, it is clear that the system of functions $\left[w^{*}, U^{*}, V^{*}\right]=$ $\left[\frac{w^{*}}{k^{*}}, \frac{U^{*}}{k^{*}}, \frac{V^{*}}{k^{*}}\right]$ is a solution of the boundary value problem

$$
\begin{gathered}
U_{\bar{z}}^{*}=Q_{1} U_{z}^{*}+Q_{2} V_{z}^{*}+A_{1} U^{*}+A_{2} V^{*}+A_{3} w^{*}+\frac{A_{4}}{k^{*}} \\
V_{\bar{\xi}}^{*}=\overline{U^{*}} z \\
\operatorname{Re}\left[\overline{\lambda_{j}(z)} U^{*}(z)+a_{j 1}(z) w^{*}(z)\right]=\frac{a_{j 2}(z)+h_{j}(z)}{k^{*}} \quad(z \in \Gamma) \\
\left.\operatorname{Im}\left[\overline{\lambda_{j}(z)} U^{*}(z)+a_{j 1}(z) w^{*}(z)\right]\right|_{z=a_{k}}=\frac{b_{j k}}{k^{*}}
\end{gathered}
$$

where $j=1,2$ and $k \in J_{j}$, and

$$
w^{*}(z)=-\int_{0}^{z}\left[\frac{U^{*}(\zeta)}{\zeta^{2}} d \zeta-\sum_{m=1}^{N} \frac{d_{m} z_{m}}{k^{*} \zeta\left(\zeta-z_{m}\right)} d \zeta+\frac{\overline{V^{*}(\zeta)}}{\bar{\zeta}^{2}} d \bar{\zeta}\right]+\frac{c_{0}}{k^{*}} .
$$

From (1.2), (1.5) and (1.12) we see that

$$
L_{p, 2}\left[\frac{A_{4}}{k^{*}}, \bar{D}\right] \leq 1, \quad C_{\alpha}\left[\frac{a_{j 2}}{k_{*}}, \Gamma\right] \leq 1, \quad \sum_{j=1,2 ; k \in J_{j}} \frac{\left|b_{j k}\right|}{k_{*}} \leq 1, \quad \frac{\left|c_{0}\right|}{k_{*}} \leq 1 .
$$

On the basis of the estimates in Theorem 2.1 , we obtain for the solution $\left[w^{*}, U^{*}, V^{*}\right]$ of the boundary value problem (2.17) - (2.20) the estimate

$$
L\left(U^{*}\right) \leq M_{8}, \quad L\left(V^{*}\right) \leq M_{8}, \quad S\left(w^{*}\right) \leq M_{9} .
$$

From the above estimates it immediately follows that estimates (2.15) and (2.16) hold 
Remark. Through the mapping $z=z(\zeta)=\frac{1}{\zeta}$ the complex equation (1.1) can be reduced to the form

$$
\left.\begin{array}{rl}
w_{\zeta \bar{\zeta}} & =G\left(z, w, w_{\zeta}, \bar{w}_{\zeta,} w_{\zeta \zeta}, \bar{w}_{\zeta \zeta}\right) \\
G & =\tilde{Q}_{1} w_{\zeta \zeta}+\tilde{Q}_{2} \bar{w}_{\zeta \zeta}+\tilde{A}_{1} w_{\zeta}+\tilde{A}_{2} \bar{w}_{\zeta}+\tilde{A}_{3} w+\tilde{A}_{4}
\end{array}\right\} \quad(z \in \widetilde{D}=\zeta(D))
$$

in which

$$
\widetilde{Q}_{j}=\frac{Q_{j} \zeta^{2}}{\bar{\zeta}^{2}}, \tilde{A}_{j}=-\frac{A_{j}}{\bar{\zeta}^{2}}(j=1,2) \quad \text { and } \quad \tilde{A}_{j}=\frac{A_{j}}{|\zeta|^{4}} \quad(j=3,4) \quad(\zeta \in \widetilde{D})
$$

and $\zeta=\zeta(z)=\frac{1}{z}$. By Condition (C), the above coefficients satisfy the conditions

$$
\left|\widetilde{Q}_{1}\right|+\left|\widetilde{Q}_{2}\right| \leq q_{0} \quad(\zeta \in \tilde{D}) \quad \text { and } \quad L_{p, 2}\left[\tilde{A}_{j}, \overline{\widetilde{D}}\right] \leq k_{j-1} \quad(j=1,2,3,4)
$$

If the function $w$ is a solution of the complex equation (1.1) with Condition (C) in $D$, then $w(z)=w[z(\zeta)]=w\left[\frac{1}{\zeta}\right]$ is a solution of the complex equation (2.22) in $\widetilde{D}$. Noting that $w_{z \bar{z}}=|\zeta|^{4} w_{\zeta \bar{\zeta}}$ and $w_{z z}=\zeta^{4} w_{\zeta \zeta}$, we see that if $w(z) \in W_{p_{0}, 4}^{2}(D) \quad\left(2<p_{0} \leq p\right)$, then $w[z(\zeta)] \in W_{p_{0}}^{2}(\tilde{D})$. The inverse result is also true.

Moreover, denoting $U(z)=U[z(\zeta)]=U\left(\frac{1}{\zeta}\right)$, we have $U_{\bar{z}}=-\bar{\zeta}^{2} U_{\bar{\zeta}}$ and $U_{z}=-\zeta^{2} U_{\zeta}$, and we see that if $U(z) \in W_{p_{0}, 2}^{1}(D) \quad\left(2<p_{0} \leq p\right)$, then $U[z(\zeta)] \in W_{p_{0}}^{1}(\widetilde{D})$. The inverse result is also true.

If $f(z) \in L_{p_{0}, 2}(\bar{D})$, then

$$
\left.\begin{array}{rl}
T f & =-\frac{1}{\pi} \iint_{D} \frac{f(\zeta)}{\zeta-z} d \sigma_{\zeta}=-\frac{1}{\pi} \iint_{\tilde{D}} \frac{f\left(\frac{1}{\zeta}\right)}{\bar{\zeta}^{2} \zeta(1-\zeta z)} d \sigma_{\zeta}=S(0)-S\left(\frac{1}{z}\right) \\
S(z) & =-\frac{1}{\pi} \iint_{\widetilde{D}} \frac{\tilde{f}(\zeta)}{\zeta-z} d \sigma_{\zeta}, \quad \tilde{f}(\zeta)=\frac{f\left(\frac{1}{\zeta}\right)}{\bar{\zeta}^{2}} .
\end{array}\right\}
$$

This shows that $\tilde{f}(z) \in L_{p_{0}}(\overline{\widetilde{D}})$. Hence

$$
\begin{array}{r}
\widetilde{C}_{\alpha}[S(z), \widetilde{D}] \leq M L_{p_{0}}[\tilde{f}(z), \overline{\widetilde{D}}] \\
\widetilde{C}_{\alpha}\left[S(0)-S\left(\frac{1}{z}\right), \tilde{D}\right] \leq M L_{p_{0}}[\tilde{f}(z), \overline{\widetilde{D}}]
\end{array}
$$

in which $\alpha=1-\frac{2}{p_{0}}$ and $M=M\left(p_{0}\right)$. Thus by using the method of continuity and the contracting mapping principle, we can prove that there exist the solutions $\psi=T f$ and $\phi=T g \in W_{p_{0}, 2}^{1}(\bar{D})$ of

$$
\begin{aligned}
& \psi_{\bar{z}}=Q_{1} \psi_{z}+A_{1} \psi+A, \quad A=Q_{2} V_{z}+A_{2} V+A_{3} w+A_{4} \\
& \phi_{\bar{z}}=Q_{1} \phi_{z}+A_{1}
\end{aligned}
$$

in $D$. Moreover, we can also find the solution $\chi(z)=\frac{1}{z}+T h$ of the equation

$$
W_{\bar{z}}=Q W_{z} \quad \text { or } h(z)=Q(z)\left[-\frac{1}{z^{2}}+\Pi h\right]
$$


It is clear that $-\frac{Q(z)}{z^{2}} \in L_{p, 2}(\bar{D})$, and then $h(z) \in L_{p_{0}, 2}(\overline{\widetilde{D}})$. Due to the fact that the function $\chi\left(\frac{1}{z}\right)=z+S(0)-S(z)=z+S(0)-T[\tilde{h}]$ is a solution of

$$
\tilde{h}(z)=\frac{\widetilde{Q}(z) z^{2}}{\bar{z}^{2}[1+\Pi \tilde{h}]} \quad \text { in } \tilde{D}
$$

where $\tilde{h}(\zeta)=\frac{h\left(\frac{1}{\zeta}\right)}{\zeta^{2}}$, the above function $\chi\left(\frac{1}{z}\right)$ is a homeomorphism in $\tilde{D}$. Obviously, $\chi(z)$ is also a homeomorphism in $\dot{D}$.

From Theorem 2.1 we sec that the solution $w=w(z)$ satisfies the estimate

$$
U(z), V(z)=O\left(|z|^{\frac{2}{p_{0}}-1}\right) \quad \text { as } \quad z \rightarrow \infty \quad \text { and } \quad \int_{\widetilde{\Gamma}}[U(z) d z+V d \bar{z}]=0
$$

where $\widetilde{\Gamma}=\{z \in \mathbb{C}:|z|=R\}$. Herein $R$ is a sufficiently large number. Hence $\dot{w}$ is in $\bar{D}$ continuously differentiable.

\section{Solvability of boundary value problems}

On the basis of proper a priori estimates nonlinear problems are often solved by the Leray-Schauder technique. This method is extensively used in $[2,3]$ for different problems. In this way here the solvability of problems (P) and (Q) are discussed.

Theorem 3.1. If Conditions $(\mathrm{C})_{1}-(\mathrm{C})_{3}$ and (1.13) hold, and the constants $q_{2}$ and $k_{1}, k_{2}$ in (1.2),(1.3) and (1.5) are small enough, then the solution $[w, U, V]$ of Problem (Q) is unique.

Proof. Denote by $\left[w^{j}, U^{j}, V^{j}\right](j=1,2)$ two solutions of Problem (Q) and substitute them into (1.7) - (1,9) and (1.11). Then $[w, U, V]=\left[w^{1}-w^{2}, U^{1}-U^{2}, V^{1}-V^{2}\right]$ is a solution of the homogeneous boundary value problem

$$
\left.\begin{array}{c}
\left.U_{\Sigma}=\tilde{Q}_{1} U_{z}+\tilde{Q}_{2} V_{z}+\tilde{A}_{1} U+\tilde{A}_{2} V+\tilde{A} w\right\} \\
V_{\bar{z}}=\tilde{U}_{z}
\end{array}\right\} \quad \begin{gathered}
(z \in \Gamma) \\
\operatorname{Re}\left[\overline{\lambda_{j}}(z) U_{j}(z)+a_{j 1}(z) w(z)\right]=h_{j}(z) \quad\left(j=1,2 ; k \in J_{j}\right) \\
\left.\left.\operatorname{Im}\left[\overline{\lambda_{j}(z)} U(z)+a_{j 1}(z) w(z)\right]\right|_{z=a_{k}}=0 \quad \frac{\overline{V(\zeta)}}{\bar{\zeta}^{2}} d \bar{\zeta}\right] \\
w(z)=-\int_{1}^{z}\left[\frac{U(\zeta)}{\zeta^{2}} d \zeta-\sum_{m=1}^{N} \frac{d_{m} z_{m}}{\zeta\left(\zeta-z_{m}\right)} d \zeta+\frac{}{\zeta(\zeta)}\right.
\end{gathered}
$$

the coefficients of which satisfy conditions (1.7) - (1.9) and (1.11), but $k_{3}=k_{4}=k_{5}=0$. On the basis of Theorem 2.2 , provided $q_{2}$ and $k_{1}, k_{2}$ are sufficiently small, we can derive that $U=V=w=0$ on $\bar{D}$, i.e. $w^{1}=w^{2}, U^{1}=U^{2}$ and $V^{1}=V^{2}$ on $\bar{D}$ 
In the following, we use the foregoing estimates of solutions and the Leray-Schauder theorem to prove the solvability of Problem (Q) for the nonlinear elliptic system.

Theorem 3.2. Suppose that the conditions of Theorem 2.1 are satisfyed. Then Problem (Q) is solvable.

Proof. First of all, we assume that $F\left(z, w, U, V, U_{z}, V_{z}\right)=0$ from (1.7) in the neighbourhood $D^{*}$ of the boundary $\Gamma$, namely

$$
\left.\begin{array}{l}
U_{\bar{z}}^{*}=t F^{*}\left(z, w, U, V, U_{z}^{*}, V_{z}^{*}\right) \\
V_{\bar{z}}^{*}=t \bar{U}_{z}^{*}
\end{array}\right\} \quad(0 \leq t \leq 1) .
$$

We introduce the Banach space

$$
B=W_{p_{0}, 2}^{1}(D) \times W_{p_{0}, 2}^{1}(D) \times C^{1}(\bar{D}) \quad\left(2<p_{0} \leq p\right) .
$$

Denote by $B_{M}$ the set of triples of continuous functions $\omega=[w, U, V]$ satisfying the inequalities

$$
\left.\begin{array}{rl}
L(U)=C_{\beta}[U, \bar{D}]+L_{p_{0}, 2}\left[\left|U_{\bar{z}}\right|+\left|U_{z}\right|, \bar{D}\right] & <M_{10} \\
L(V) & <M_{10} \\
C^{1}[w(z), \bar{D}] & <M_{11}
\end{array}\right\}
$$

where $M_{10}=M_{1}+1$ and $M_{11}=M_{2}+1$, with $\beta$ and $M_{1}, M_{2}$ being non-negative constants as stated in (2.1) and (2.2). It is cvident that $B_{M}$ is a bounded open set in $B$.

Next, we arbitrarily select a system of functions $\omega=[w, U, V] \in B_{M}$ and substitute it into the appropriate positions of (1.7) - (1.9) and (1.11), and then consider the boundary value problem $(\mathrm{Q})^{\prime}$ with parameter $t \in[0,1]$

$$
\begin{aligned}
& \left.\begin{array}{l}
U_{\bar{z}}^{*}=t F^{*}\left(z, w, U, V, U_{z}^{*}, V_{z}^{*}\right) \\
V_{\bar{z}}^{*}=t \bar{U}_{z}^{*}
\end{array}\right\} \quad \text { in } D \\
& \operatorname{Re}\left[\overline{\lambda_{j}(z)} U^{*}(z)+t a_{j 1}(z) w(z)\right]=a_{j 2}(z)+h_{j}(z) \quad(z \in \Gamma) \\
& \left.\operatorname{Im}\left[\overline{\lambda_{j}(z)} U^{*}(z)+t a_{j 1}(z) w(z)\right]\right|_{z=a_{k}}=\dot{b}_{j k} \quad\left(j=1,2 ; k \in J_{j}\right) \\
& w^{*}(z)=-\int_{0}^{z}\left[\frac{U^{*}(\zeta)}{\zeta^{2}}-\sum_{m=1}^{N} \frac{d_{m} z_{m}}{\zeta\left(\zeta-z_{m}\right)}\right] d \zeta+\frac{\overline{V^{*}(\zeta)}}{\bar{\zeta}^{2}} d \bar{\zeta}
\end{aligned}
$$

where $w, U, V$ are known functions as stated beforc. Noting that Problem (Q) consists of two modified Riemann-Hilbert boundary value problems for elliptic complex equations of first order and applying [4: Theorem 3.2], there exist the solution $U^{*}, V^{*} \in$ $W_{p_{0}, 2}^{1}(D) \quad\left(2<p_{0} \leq p\right)$. From $(3.10)$, the single-valued function $w^{*}$ on $\bar{D}$ is determined. Denote by $\omega^{*}=\left[w^{*}, U^{*}, V^{*}\right]=T(\omega, t)(0 \leq t \leq 1)$ this mapping from $\omega$ onto $\omega^{*}$. According to Theorem 2.1 , if $\omega=[w, U, V]=T(\omega, t)$, then $\omega=[w, U, V]$ satisfies estimates 
(2.1) and (2.2), consequently $\omega \in B_{M}$. Setting $B_{0}=B_{M} \times[0,1]$, we shall verify that the mapping $\omega^{*}=T(\omega, t)$ satisfies the three conditions of the Leray-Schauder theorem:

(1) When $t=0$, by Theorem 2.1, it is evident that $\omega^{*}=T(\omega, t) \in B_{M}$.

(2) As stated before, the solution $\omega=[w, U, V]$ of the functional equation $\omega=$ $T(\omega, t)$ satisfies estimates (2.1) and (2.2) which shows that $\omega=T(\omega, t)$ does not have a solution $\omega=[w, U, V]$ on the boundary $\partial B_{M}=\overline{B_{M}} \backslash B_{M}$.

(3) $\omega^{*}=T(\omega, t)$ continuously maps the Banach space $B$ into itself, and is completely continuous on $B_{M}$. Besides, for $\omega \in \overline{B_{M}}, T(\omega, t)$ is uniformly continuous with respect to $t$.

In fact, let us choose any sequence $\left\{\omega_{n}\right\}_{n \in N}=\left\{\left[w_{n}, U_{n}, V_{n}\right]\right\}_{n \in N} \subset \overline{B_{M}}$. By Theorem 2.1 , it is not difficult to see that $\omega_{n}^{*}=\left[w_{n}^{*}, U_{n}^{*}, V_{n}^{*}\right]=T\left(\omega_{n}, t\right)(0 \leq t \leq 1)$ satisfies the estimates

$$
L\left(U_{n}^{*}\right) \leq M_{12}, \quad L\left(V_{n}^{*}\right) \leq M_{12}, \quad S\left(w_{n}^{*}\right) \leq M_{13}
$$

where $M_{j}=M_{j}\left(q_{0}, p_{0}, k, \alpha, K, D, M\right)(j=12,13)$. Hence there can be selected subsequences of $\left\{w_{n}^{*}\right\},\left\{U_{n}^{*}\right\}$ and $\left\{V_{n}^{*}\right\}$, which uniformly converge to $w_{0}^{*}, U_{0}^{*}$ and $V_{0}^{*}$ on $\bar{D}$, and $\left\{U_{n z}^{*}\right\},\left\{U_{n \bar{z}}^{*}\right\}$ and $\left\{V_{n z}^{*}\right\},\left\{V_{n \bar{z}}^{*}\right\}$ in $D$ weakly converge to $U_{0 z}^{*}, U_{0 \bar{z}}^{*}$ and $V_{0 z}^{*}, V_{0 \bar{z}}^{*}$, respectively. For convenience, denote by the same symbols as before these subsequences. From $\omega_{n}^{*}=T\left(\omega_{n}, t\right)$ and $\omega_{0}^{*}=T\left(\omega_{0}, t\right)(0 \leq t \leq 1)$ we obtain

$$
\begin{aligned}
U_{n \bar{z}}^{*}-U_{0 \bar{z}}^{*}= & t\left[F\left(z, w_{n}, U_{n}, V_{n}, U_{n z}^{*}, V_{n z}^{*}\right)\right. \\
& \left.-F\left(z, w_{n}, U_{n}, V_{n}, U_{0 z}^{*}, V_{0 z}^{*}\right)+c_{n}\right] \\
c_{n}= & F\left(z, w_{n}, U_{n}, V_{n}, U_{0 z}^{*}, V_{0 z}^{*}\right)-F\left(z, w_{0}, U_{0}, V_{0}, U_{0 z}^{*}, V_{0 z}^{*}\right) \\
V_{n \bar{z}}^{*}-V_{0 \bar{z}}^{*}= & t\left[\bar{U}_{n z}^{*}-\bar{U}_{0 z}^{*}\right]
\end{aligned}
$$

It is not difficult to see that $c_{n} \rightarrow 0$ for almost every point $z \in D$ as $n \rightarrow \infty$. Hence we can prove that $L_{p_{0}}\left[c_{n}, \bar{D}\right] \rightarrow 0$ for $n \rightarrow \infty$ as follows: Choosing two arbitrary sufficiently small positive constants $\varepsilon_{1}$ and $\varepsilon_{2}$, there exist a subset $D, \subset D$ and a sufficiently large positive integer $N$ such that meas $D_{*}<\varepsilon_{1}$ and $\left|c_{n}\right|<\varepsilon_{2}$ on $\bar{D} \backslash D_{*}$ for $n>N$. By the 
Hölder and Minkowski inequalities we have

$$
\begin{aligned}
L_{p_{0}, 2}\left[c_{n}, \bar{D}\right] & \leq L_{p_{0}, 2}\left[c_{n}, D_{*}\right]+L_{p_{0}, 2}\left[c_{n}, \bar{D} \backslash D_{*}\right] \\
& \leq L_{p_{1}, 2}\left[c_{n}, D_{*}\right] L_{p_{2}, 2}\left[1, D_{*}\right]+\varepsilon_{2} L_{p_{0}, 2}\left[1, \bar{D} \backslash D_{*}\right] \quad \\
& \leq M_{14} \varepsilon_{1}^{1 / p_{2}}+\varepsilon_{2} \pi^{1 / p_{0}} \\
& =\varepsilon
\end{aligned}
$$

where $p_{2}=\frac{p_{0 p_{1}}}{p_{1}-p_{0}}, 2<p_{0}<p_{1}<p_{2}<\infty$ and $M_{14}$ is a non-negative constant. On the basis of Theorem 2.2, it can be derived that

$$
\left.\begin{array}{r}
L\left(U_{n}-U_{0}\right) \\
L\left(V_{n}-V_{0}\right) \\
S\left(w_{n}-w_{0}\right)
\end{array}\right\} \rightarrow 0 \quad(n \rightarrow \infty)
$$

Because of the completeness of the Banach space $B$, there exists a system of functions $\omega_{0}=\left[w_{0}, U_{0}, V_{0}\right] \in B$ such that

$$
\left.\begin{array}{r}
L\left(U_{n}-U_{0}\right) \\
L\left(V_{n}-V_{0}\right) \\
S\left(w_{n}-w_{0}\right)
\end{array}\right\} \rightarrow 0 \quad(n \rightarrow \infty)
$$

This shows the complete continuity of $\omega^{*}=T(\omega, t)(0 \leq t \leq 1)$ on $\overline{B_{M}}$. By a similar method we can also prove that $\omega^{*}=T(\omega, t)$ continuously maps $\overline{B_{M}}$ into $B$ and $T(\omega, t)$ is uniformly continuous with respect to $t$ for $\omega \in \overline{B_{M}}$.

Hence by the Leray-Schauder theorem, we see that the functional equation $\omega=$ $T(\omega, t) \quad(0 \leq t \leq 1)$ with $t=1$, i.e. Problem (Q) has a solution.

Finally, we can eliminate the assumption of $F\left(z, w, U, V, U_{z}, V_{z}\right)=0$ in $D^{*}$ and prove the solvability of Problem (Q) for the general nonlinear elliptic system (1.7) in $D$. This completes the proof

Theorem 3.3. Under the same conditions as in Theorem 3.2, the result of solvability of Problem (P) for the complex equation is as follows:

(1) If $K_{n}=\frac{1}{2 \pi} \Delta_{\Gamma} \arg \lambda_{n}(z) \geq N$ ( $\left.n \in \mathbb{N}\right)$, then Problem (P) has $2 N$ solvability conditions, and the general solution depends on $2\left(K_{1}+K_{2}-2 N+2\right)$ arbitrary real constants.

(2) If $0 \leq K_{j}<N \quad(j=1,2)$, the total number of solvability conditions of Problem (P) is not greater than $4 N-K_{1}-K_{2}$, and the general solution depends on $K_{1}+K_{2}+4$ arbitrary real constants.

(3) If $K_{j}<0 \quad(j=1,2)$, then Problem (P) has $4 N-2 K_{1}-2 K_{2}-2$ solvability conditions, and the general solution depends on two real constants.

We can also write solvability conditions of Problem (P) in other cases.

Proof. We only discuss the case $0 \leq K_{j}<N(j=1,2)$. Let the sölution $[w, U, V]$ of Problem (Q) be substituted into (1.7) - (1.9) and (1.11). The functions $h_{j}(j=1,2)$ 
and the complex constants $d_{m}(m=1, \ldots, N)$ are then determined: If the functions and the constants are equal to zero, namely

$$
h_{j}(z)=h_{j k}\left(j=1, \ldots, N-K_{j}\right) \quad \text { when } 0 \leq K_{j}<N \quad(j=1,2)
$$

and

$$
d_{m}=0 \quad(m=1, \ldots, N)
$$

then $w_{z}=U(z)$ and $\bar{w}_{z}=V(z)$, and $w$ is a solution of Problem (P). Hence when $0 \leq K_{j}<N(j=1,2)$, Problem (P) has $4 N-K_{1}-K_{2}$ solvability conditions. In addition, the real constants $b_{j k}\left(k=N-K_{j}+1, \ldots, N+1 ; j=1,2\right)$ in (1.11) and the complex constant $c_{0}$ in (1.9) may be arbitrary. This shows that the general solution of Problem (P) $\left(0 \leq K_{j}<N_{;} j=1,2\right)$ depends on $K_{1}+K_{2}+4$ arbitrary real constants

\section{References}

[1] Vekua, I. N.: Generalized Analytic Functions. Oxford: Pergamon Press 1962.

[2] Wen, G. C. and H. Begehr: Boundary Value Problems for Elliptic Equations and Systems. Harlow: Longman Sci. and Techn. 1990.

[3] Begehr, H. and G. C. Wen: Nonlinear Elliptic Boundary Value Problems and Their Applications. Harlow: Longman Sci. and Techn. 1996.

1996.

[4] Wen, G. C.: The Riemann-Hilbert boundary value problem for nonlinear elliptic complex equations of first order in multiply connected infinite domains. In: Partial Differential and Integral Equations (eds.: H. Begehr, R. P. Gilbert and G.-C. Wen). Dordrecht: Kluwer 1999 , pp. $85-95$.

[5] Wolfersdorf, L. von: On the theory of nonlinear generalized Poincare problems for harmonic functions. In: Continuum Mechanics and Related Problems of Analysis (eds.: $M$. Balavadze et al.). Tbilisi: Metsniereba 1993, pp. $330-337$. 